\title{
Development of Continuing Professional Development (CPD) Provision Framework for Sri Lankan Grade Medical Officers
}

\author{
Jayarathne Y.G.S. $W^{1}$, Karunathilake, I.M ${ }^{2}$, Marambe, K.N ${ }^{3}$
}

\begin{abstract}
Introduction: CPD activities are provided by various organizations in Sri Lanka and a wide variation is observed. However, it is crucial to have a common CPD framework to be utilized by every CPD organizing body.
\end{abstract}

Objective: The aim of this study is to develop a CPD provision framework and CPD provision model for Sri Lankan grade medical officers.

Methodology: A conceptual model was constructed to enable the effective CPD provision to Grade Medical Officers (GMOs) in Sri Lanka, using baseline information on lifelong learning and CPD practices of GMOs in the Central Province and views from island wide CPD leads. Subsequently, a 15 item CPD provision - framework and the conceptual model was developed. As a next step, feasibility to implement the proposed framework was assessed among the selected CPD leads through a semi- structured interview. Finally, the modified CPD provision framework and the CPD provision model for Sri Lanka were finalized.

Results: In the CPD provision model, Physicians, CPD providers and Accreditation bodies were identified as key responsible entities while identification of learning needs, proper planning, mechanism for action and evaluation were identified as main steps for effective CPD provision. CPD leads were unanimous on the need for structured CPD programs. Eight of 15 items in the proposed CPD provision framework were accepted by all the interviewed CPD leads.

Conclusions: Since the draft version of the CPD provision framework received high level of acceptance, the Ministry of Health should take the lead in developing it further as well as take measures to regularize CPD among doctors.

Key Words: CPD, CME, CPD Models, CPD Programmes

\section{Introduction}

In Sri Lanka, CPD activities are provided by various organizing bodies and a wide variation is seen among the CPD programmes conducted by them. However, it is essential to have a commonality between CPD programmes offered in a given country and it should be paralleled to the international CPD systems. Such a move would enable the CPD programmes to be recognized by other responsible bodies overseas. Thus, it is crucial to have a common CPD structure for every CPD organizing body.

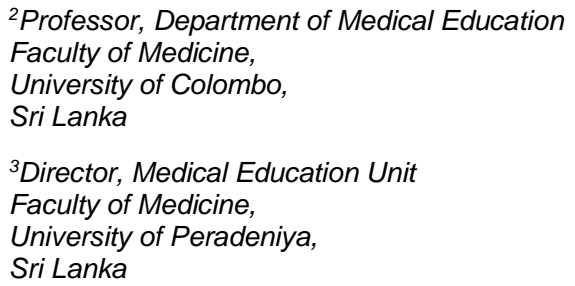

Corresponding Author:

${ }^{1}$ Dr. Y.G.S.W.Jayarathne Lecturer in Medical Education, Faculty of Medicine,

Rajarata University of Sri Lanka

Email:wjayarathne@yahoo.com 
It is evident from the literature, that participation in high quality CPD programmes improves clinical practice and health care outcomes. Thus, CPD providers have a bounding responsibility to play a leading role in defining, designing and implementing high quality CPD programmes to enhance physicians' performance (Bennett, et al, 2000). It is also well known that in many developed and developing countries, CME/CPD programmes are evolving rapidly either on a voluntary or mandatory basis. Also, many countries have gone to the extent of revising regulations to enhance professional development of doctors. However, the current situation in Sri Lanka is once qualified and registered, a doctor is licensed to practice for life and there are no policies in relation to the CPD of doctors. Further, currently CPD provision guidelines are not available in Sri Lanka. Hence, the outcome of the overall study was to develop a CPD provision framework for Sri Lankan GMOs which could be adopted to streamline CPD provision in Sri Lanka.

\section{Methodology}

In order to develop a CPD provision framework for Sri Lankan GMOs, a conceptual model for an effective CPD system was developed, based on identified important aspects in literature, using integrated results of the questionnaire study (Study 1) carried out with the GMOs in the Central Province and the discussions with CPD organizing bodies (Study 2) in Sri Lanka. The study 1 involved collecting data from GMOs in the Central Province, of Sri Lanka. The probability proportional to the size, multistage sampling procedure was used to select the 422 GMOs from the different health care institutes in the Central Province. Information on lifelong learning practices (Preferred learning methods, practiced learning methods, lifelong learning preferences, learning needs) and CPD awareness and perceptions of GMOs (knowledge and understanding of concept of CPD, CPD provider, National CPD Certificate, about CPD activities and about motivators and inhibitors for CPD attendance) were obtained.

(Preferred learning methods refer to the best ways of learning as indicated by the physicians among the many methods available to them. Practiced learning methods refer to recently practiced learning methods; it could be the best learning methods or the methods selected by the physician based on available resources, which suit the immediate learning need.)
The second component of the project (Study 2) involved an island wide interview study among majority CPD leads to identify the current process of organizing CPD activities. Semistructured interviews were conducted through both telephone and face to face. Frequencies were calculated for quantitative data and qualitative data were analysed by means of content analysis.

As a next step, a 15 item CPD provision framework was developed based on the emerged conceptual model. Then, feasibility to implement the proposed framework was assessed among the selected CPD stakeholders in Sri Lanka through a semi structured interview. Finally, the modified CPD provision framework and the CPD provision model for Sri Lanka were developed based on views of interviewed CPD leads.

Ethical approval for the study was taken from Ethics review committee, Faculty of Medicine, University of Peradeniya.

\section{Results}

Based on integrated results of Study 1 and study 2, main aspects for establishing an effective CPD process for Sri Lankan GMOs were identified as follows: CPD learning needs, learning preferences, preferred CPD activities, accreditation body, CPD evaluation system, resources, an action plan and notification system, reward system, organizing structure, CPD competencies and CPD activity assessment structure. A closer observation of the identified concepts, enabled categorization of these concepts into three key areas: Physicians, CPD providers and Accreditation bodies. Further, main steps for effective CPD provision was identified as identification of learning needs, proper planning, having a proper mechanism for action and evaluation of CPD.

A 15 item CPD provision framework is illustrated in Figure 1. 
Figure 1: CPD provision framework

Item No 01- CPD organizational structure

Overall policy maker

Governing Body

National level CPD

Committee (national

level CPD planner)

\section{Provincial level CPD}

Committee (regulatory body

from national level to provincial

level and between different

clinical societies)

\section{Institutional level CPD}

Committee (institutional level CPD planner)
SLMC

Ministry of Health
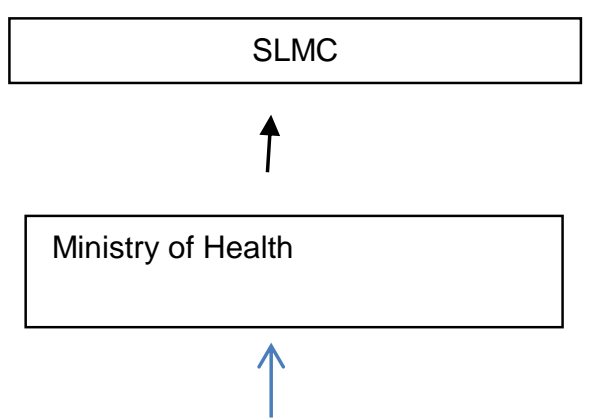

Members from SLMA, GMOA ,Ministry of Health, Provincial committee ,Other CPD organizing committees, Medical educationists

Organizations which provide CPD at national level - Composition will vary depending on the organization. However, medical educationists and GMOs need to be included.
Members from CPD committees of Clinical societies, Associations, ET \& $R$ at provincial level, Medical educationists

\section{个}

Consultants from different disciplines, GMOs of institute, GMOs from peripheral hospitals, Director, Medicaleducationist

Item No 02 - CPD committee should be composed of a consultant from each discipline, Director or Medical Superintendent of the hospital, grade medical officers, a few medical officers from peripheral hospitals and medical educationists. The CPD committee should meet monthly and the progress of activities should be discussed.

Item No 03 - CPD activities should aim at enhancing the five competencies shown below (CPD competencies). Such a strategy will no doubt enable the medical professionals to be up to date and confident. Thus, as a result, the quality of care offered by the national health system will be enhanced 
Figure 1: CPD provision framework Continue

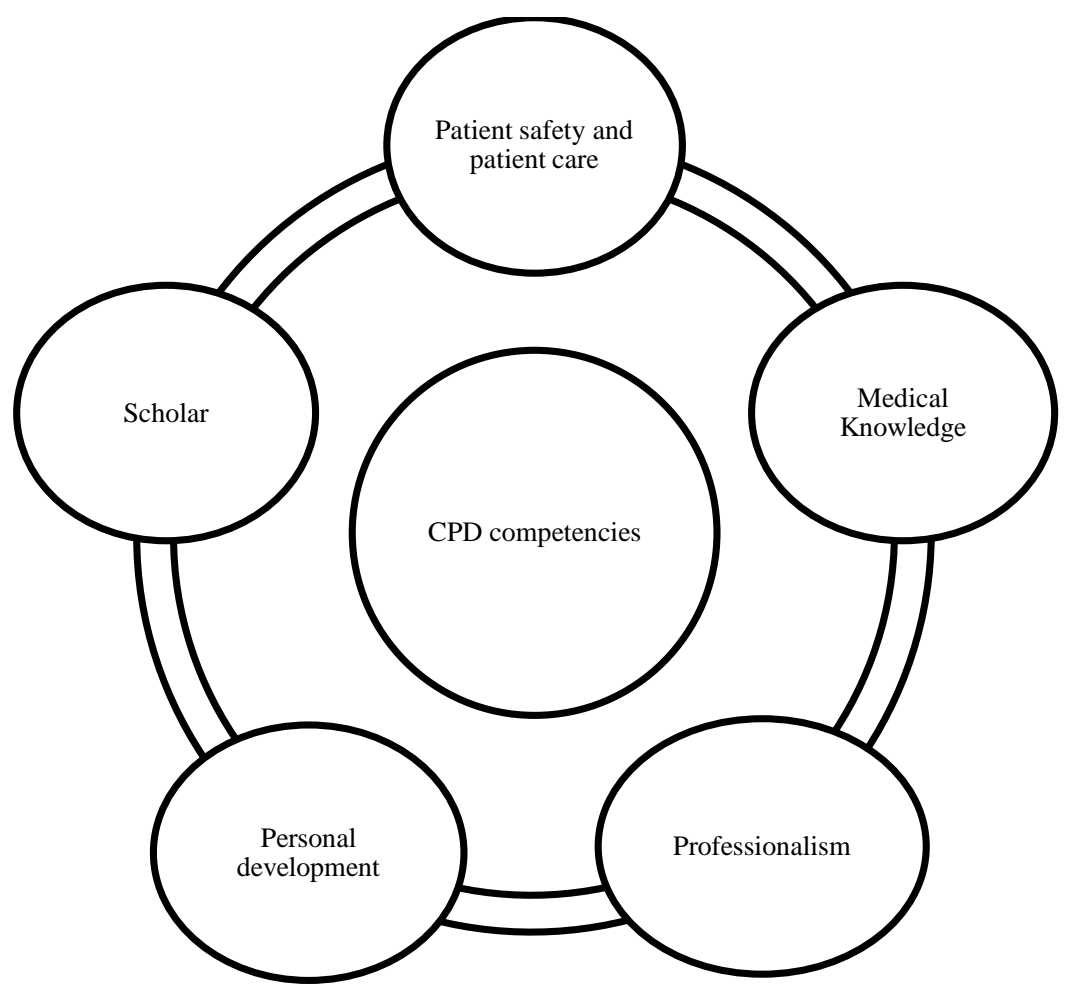

Item 3 CPD competencies

Patient safety and patient care - be competent in emergency management and routine management Medical knowledge - update the knowledge in all the fields of medicine

Professionalism - maintain ethical standards and desirable attitudes

Personal development - changing the behavior as a professional, acquiring good interpersonal skills and communication skills

Scholar - emphasis on four main concepts such as lifelong learning/CPD, critical appraisal, teaching and researching

Item No 04 - Annual CPD activity plan should be prepared to cover above all five competencies.

Item No 05 - The content area should be decided according to the relevance to practice (based on needs of national, local and institutional) and needs of individual doctors under competency domains described above.

Item No 06 - Intended learning outcomes need to be documented for each CPD activity and these activities should be arranged based on these objectives / outcomes. 


\section{Figure 1: CPD provision framework Continue}

Item No 07 - It is important to utilize the immediately available learning resources through promoting self-directed learning activities such as reading, e-learning and interactive learning (discussion with colleagues and seniors).

Item No 08 - A variety of CPD activities should be organized by the CPD committees. These activities need to be organized as longitudinal activities. Possible activities need to be started within the functional unit as such a mechanism is likely to address the most relevant needs required to improve day to day practice. Whenever possible case based interactive activities need to be incorporated in to formal CPD activities such as clinical meetings, workshops, collaborative workshops, and journal clubs.

Item No 09 - Since participation in CPD activities is voluntary, incorporating structured systematic learning in to work based activities and participation of CPD activities in duty rosters will be more beneficial. (Main reasons for poor participation in CPD mentioned were heavy workload, lack of motivation, and difficulties encountered in getting leave). Thus, it is important to consider the consultant in-charge of the unit as the leader in improving work based learning activities.

Item No 10 - Library facilities, especially e-learning facilities should be arranged by the hospital. And higher authorities need to be attentive on providing resources (physical resources, updated guidelines, free access to evidence based data bases and journals) for CPD activities of grade medical officers. Especially, available funding facilities need to be informed to the CPD organizing committees in advance by the higher authorities in order to utilize the available funding.

Item No 11 - Appraisal of the CPD activities of each hospital /CPD organizing body should be done by medical education units of regional medical faculties or education and research unit of the Ministry of Health.

Item No 12 - CPD activities should be notified well in advance. The notices should be distributed to each and every ward. It should be placed in a specific CPD notice board in each ward. It is advisable to send the CPD activity plan of major hospitals to close by peripheral hospitals, $\mathrm{MOH}$ offices and PMCU and if possible notices should be posted in the web site. (Notices should be sent to the hospitals and relevant units by other CPD organizing bodies). 


\section{Figure 1: CPD provision framework Continue}

Item No 13 - CPD organizers should motivate all the doctors to participate in regular CPD activities. Provision of incentives is important to improve the participation. e.g. - CPD certificates, a CPD based reward system, refreshments etc.

Item No 14 - All the grade medical officers should be educated regarding the value of the National CPD Certificate.

Item No 15 - CPD committee should get feedback after each activity and comments should be considered when planning future activities. CPD activities should be evaluated at least at level 1 of Kirkpatrick's model on program evaluation (degree of participation and satisfaction of participants) at present and evaluation should be done at level 2 of Kirkpatrick's model in future.

As a final outcome "A CPD provision model for Sri Lanka" (depicted by Figure 2) which was the summarized version of CPD provision framework is presented.

Figure 2: A CPD provision model for Sri Lanka”

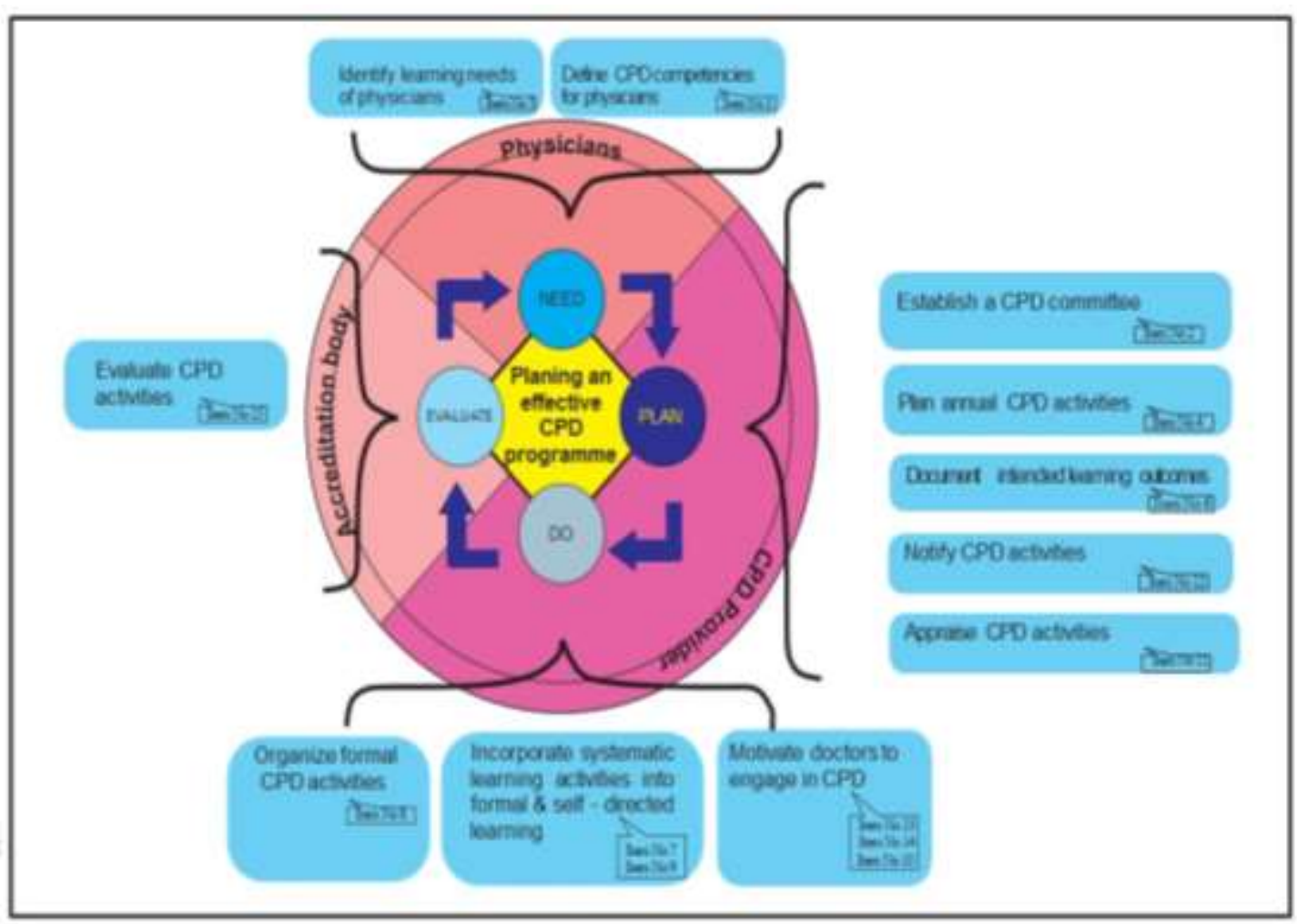




\section{Discussion:}

The aim of the proposed framework is to ensure a system of effective CPD provision to Sri Lankan GMOs. Thus, by providing a framework, it is expected that planning of CPD activities by the CPD providers will be more systematic. It is also felt that if CPD providers adopt all the strategies described in the proposed CPD provision framework, it would immensely enhance CPD opportunities for most of the doctors and participation is likely to be high. The importance of having a 15 item framework for effective CPD provision is discussed in the section that follows.

\section{CPD provision framework:}

It is believed that there should be a better functioning structure in order to operate an effective CPD program in a given country. In countries such as UK, Australia, New Zealand and Singapore, CPD standards and policies are regulated either by the Ministry of Health or Medical Councils (GMC, 2012; MBA, 2014; MCNZ, 2014; SMC, 2014). However, policies in terms of CPD of doctors in Sri Lanka are yet to be developed. Furthermore, both GMOs and CPD leads view that, the Ministry of Health, which is the main employer of doctors in Sri Lanka should be responsible and should play a major role in ensuring CPD among doctors. Therefore, in keeping with the international models, and with the suggestions of CPD leads and GMOs, Ministry of Health was selected as the governing body of CPD for Sri Lankan doctors. According to the current Sri Lankan situation, it is true that Sri Lanka Medical Association (SLMC) is the overall policy maker in regulating undergraduate training, postgraduate training and CPD of doctors. Therefore, SLMC was placed at a higher level than the Ministry of Health. Further, the middle level coordination between institutional level and national level are essential to have better functioning of the organizing structure. In addition, institutional level CPD committees will have to be established in the hospitals where there are existing clinical societies. The composition of a CPD committee also seems a decisive factor. Since planning has to be need based and educationally sound it is important to have consultants from each discipline, GMOs from the hospital as well as from the peripheral hospitals, administrative officers and a medical educationist. Hence, the planning of functioning organizing structure for CPD of a given country is important for effective CPD system.

The global standards for continuum of medical education involving undergraduate, postgraduate and continuous learning has been described by the World Federation for Medical Education, in the year 2003 (WFME, 2003). The competencies described for postgraduate and CPD are the same; however, the gravity and complexity of descriptors increase along with the qualifications. It is noted that overlapping of proposed CPD competencies (Patient safety and patient care, Medical knowledge, Professionalism, Personal development and Scholar) with the outcomes of the National CPD Certificate, subject bench mark statement of medicine and the Sri Lanka Qualification Framework (SLQF) (MHE, 2015). Thus, it is reasonable to agree on proposed competencies as the starting point of drawing up CPD provision strategies in Sri Lanka as this number seems to be manageable. However, it is felt that there is a need, to have a common competency framework for advancing the complexity of descriptors in terms of continuum of medical education in the future.

Although it is challenging, it is important to identify the learning needs of individual physicians and expected learning outcomes, in order to conduct an effective CPD programme. It is reported in the literature that failing CPD systems in some countries is due to the absence of need based accredited CPD programmes (WHO, 2010). In addition, WHO, 2010 reports that CPD programmes should be based on country's needs, perceptions and prevailing practices. It was proven that learning need analysis leads to change in physician performance (Norman et al, 2004; Oxman et al, 1995). It is also clear, if CPD activities are not relevant to the practice and not relevant to their own needs, no doctor will participate in CPD programmes.

Literature reports that, if CPD activities are used appropriately, it can improve professional practice and patient care outcomes (Kolb \& Fry, 1975). It seems appropriate to refer to theories of adult learning at this stage. According to the Kolb's Learning Theory, learners have a preferred learning style and effective learning will take place only if the preferred learning styles are 
used (Knowles, 1984). Furthermore, Knowles in 1984 stated that adults prefer problem centered learning (Stephens et al, 2011). It is also known that retention of knowledge with practice change can be achieved mainly through interactive sessions (Stephens et al, 2011; Cauffman et al, 2002; Davis, 1998a; Davis, 1998b). Furthermore, Davis and colleagues have reported that if CPD activities are systematic and practice based, effectiveness on changing physician performance is more (Davis et al, 1995). Therefore, when catering programs to a diverse group of adult learners, one needs to offer a variety of CPD activities by means of a variety of learning methods to obtain better results. Considering the preferred learning methods and also considering difficulty of releasing medical officers to participate in formal CPD activities, due to heavy workload and lack of staff, it was felt that it is better to start CPD activities within the unit. On the other hand, if CPD activities occur within the unit, the conducted activities will be relevant to practice. In addition, in the respective place of work, doctors can interact with colleagues and consultants promoting peer learning and active engagement in the learning process. Reflection on one's own experience can be done as a group and in this case provision of feedback will be easy. In addition, CPD is a voluntary act in Sri Lanka, at present. Thus, incorporating work based learning activities and incorporating CPD activities in duty rosters are important to promote lifelong learning among GMOs. Thus, CPD providers need to plan a variety of CPD activities having a system to integrate work place based learning among GMOs without further delay.

It was revealed that lack of resources such as library facilities, e-library facilities are common problems faced by most of the CPD providers. However, noteworthy is the fact that Sri Lanka has a well-developed telecommunication network which can be capitalized to promote CPD among doctors. It will be a cost effective option even for the clinical societies to organize web based seminars and case discussions. Thus, improving facilities and resources particularly, telecommunication facilities to promote technology enhanced learning by the higher authorities is vital towards establishing successful CPD programmes in Sri Lanka.

Appraisal and accreditation of CPD activities are very important to improve the quality of the conducted CPD activities. Evidence shows that most of the countries have accreditation bodies to ensure the quality of CPD activities. e.g. ACCME in USA and EACCME in Europe (ACCEME, 2012; Farrow, 2012). However, Sri Lanka is yet to develop a systematic CPD system. Applying accreditation to the framework is not practical at the moment and it was felt that at least appraisals of CPD activities are important in the present context. Since medical education units are available at all the medical faculties in Sri Lanka and education and training units are available at the Ministry of Health and provincial level, it is possible to involve these education units in the appraisal of CPD activities.

It is reported in the literature that one important reason for failing CPD systems in developing countries is lack of incentives (WHO, 2010). Schaffer, in 2000 reported that participation of CPD activities improved because of reward systems such as free lunch or other gifts in the USA (Schaffer, 2010). It is also evident that reward systems were implemented in most of the countries at the initial stages of introducing CPD systems (Kelman, 1958; Afshar, 2012). It is the human nature that people are driven by rewards. Therefore, to change existing practices among doctors, an appropriate reward should be offered, in the given case it would be desirable to work on a system of career advancement. Therefore, in order to institutionalize CPD it is imperative that the higher authorities take necessary actions to implement an acceptable reward system, without further delay.

At this stage it must be mentioned that the National CPD Certificate is awarded based on a well-organized structure for documenting CPD activities. It is evident that portfolio is the commonest method of documenting CPD in most of the counties such as UK, New Zealand and Australia (GMC, 2012; MCNZ, 2014; AMC, 2015). Selecting a portfolio as the learning and assessment tool is educationally sound, as it promotes self-directed learning and reflective practice among doctors while ensuring a high degree of relevance to day today work. Hence, promotion of the National CPD Certificate, which is the only existing format for evaluation of one's CPD status, is vital in the present Sri Lankan context.

Evaluation is essential to enhance the effectiveness of CPD programmes. Considering practical difficulties in evaluating CPD 
programmes beyond level 1 of Kirkpatrick's model (dealing with learners' satisfaction and perceptions), at the initial stages, it is desirable for the CPD organizers to evaluate learners' satisfaction on CPD content and delivery methods in Sri Lanka (Kirkpatrick, 1994). Yet, it would be desirable to extend the evaluation at least to level 2 of the Kirkpatrick's model in the future.

\section{Conclusion:}

The piloted draft version of the CPD provision framework showed high acceptance. All the CPD providers who participated in the interviews, agreed with most of the framework items and due to the fact it covered most of the guiding principles described in literature, it is reasonable to conclude that the developed items are appropriate for the present Sri Lankan context. Finally, it is expected that the CPD provision model consisting of three key areas and four stages of implementation will be attractive to policy makers who are planning to establish a national CPD system. Hence, Ministry of Health should take the lead in developing it further and also take measures to regularize CPD among doctors as a step towards ensuring patient safety.

\section{Acknowledgement:}

We would like to extend our thanks to our study participants for participating in this study.

\section{References:}

Accreditation Council for Continuing Medical Education (ACCEME). Accreditation requirements of the Accreditation Council for Continuing Medical Education. (2012) $<$ https://medicine.utah.edu/cme/forms/accme criteria.pdf> [accessed 04.02.2013].

Afshar, E. S. (2012) Financial Incentives for Improving the Quality of Primary Care, American Family Physician, 85, 7, pp 690-691.

Australian Medical Council (AMC). Standards for assessment and accreditation of specialist medical programs and professional development programs by the Australian Medical Council. (2015) <http://www.amc.org.au/files/2c1fb12996b0f6e 6e5cb5478dde9d9e991409359_original.pdf > [ accessed 02.12.2015].

Bennett, N. L., Davis, D. A., Easterling, W. E., Friedmann, P., Green, J. S., Koeppen, B. M., Mazmanian, P. E. \& Waxman, H. S. (2000) Continuing medical education: a new vision of the professional development of physicians, Academic Medicine, 75,12, pp 1167-72.

Cauffman, J. G., Forsyth, R. A., Clark, V. A., Foster, J. P., Martin, K. J., Lapsys, F. X. \& Davis, D. A. (2002) Randomized controlled trials of continuing Medical Education: what makes them most effective?, The Journal of Continuing Education in the Health Professions, 22, 4, pp 214-21.4.

Davis, D. (1998a) Continuing Medical education, global health, global learning, British Medical Journal, 316, 7128, pp 385-389.

Davis, D. (1998b) Does CME work? An analysis of the effect of educational activities on physician performance or health care outcomes, International Journal of Psychiatry in Medicine, 28, 1, pp 21-39.

Davis, D. A., Thomson, M. A., Oxman, A. D. \& Haynes, R. B. (1992) Evidence for the Effectiveness of CME, A Review of 50 Randomized Controlled Trials, Journal of the American Medical Association, 268, 9, pp 1111-1117.

Davis, D. A., Thomson, M. A., Oxman, A. D. \& Haynes, R. B. (1995) Changing Physician Performance: A Systematic Review of the Effect of Continuing Medical Education Strategies. Journal of the American Medical Association, 274, 9, 700-705.

Farrow, S., Gillgrass, D., Pearlstone, A., Torr, J. \& Pozniak, E. (2012) Setting CME standards in Europe: guiding principles for medical education, Current Medical Research \& Opinion, 28, 11, pp 1-11.

General Medical Council. Continuing professional development. Guidance for all doctors.(2012)<http://www.gmcuk.org/Continui ng_professional_development_guidance_fo r_all_doctors_0612.pdf_56438625.pdf> [accessed on August 10,2014]. 
Grant, J. (2002) Learning need assessment: assessing the need, British Medical Journal, 324, 7330, pp 156-9.

Kelman CH. (1958) Compliance, identification and internalization three process of attitudinal change, Journal of Conflict Resolution, 2, pp $51-60$.

Kirkpatrick, D.L (1994) Evaluating training programmes The four levels. San Francisco: Berrett -Koehler.

Knowles, M. S. (1984) The Adult Learner: A Neglected Species. 3rd Ed. Houston, TX: Gulf Publishing.

Kolb. D. A. \& Fry, R. (1975) Toward an applied theory of experiential learning in C. Cooper ed. Theories of Group Process. London: John Wiley.

Medical Board of Australia. Registration standards. (2014)

<http://www.medicalboard.gov.au/Registration -Standards.aspx> [accessed on August 01. 2014].

Medical Council of New Zeeland. Recertification and professional development. (2014)https://www.mcnz.org.nz/maintainregistration/recertification-and-professionaldevelopment/ [accessed on August 10. 2014].

Ministry of Higher Education. Sri Lanka Qualifications Framework (SLQF), Updated Version - February 2015. (2015) <http://www.qaacouncil.lk/publications/SLQF_ 13\%20May\%202015.pdf > [accessed on 15. 2015].

Norman, G. R., Shannon, S. I. \& Marrin, M. L. (2004) The need for needs assessment in continuing medical education, British Medical Journal, 328, 7446, pp 999-1001.

Oxman, A. D., Thomson, O. M. A., Davis, D. A. \& Haynes, R. B. (1995) No Magic Bullets: a systematic review of 102 trials of interventions to improve professional practice, Canadian Medical Association Journal, 153, 10, pp 14231431.
Schaffer, M. H. (2000) Commercial Support and the Quandaryof Continuing Medical Education, The Journal of Continuing Education in the Health Professions, 20, 2, pp 120-126.

Singapore Medical council. Information for registered doctors. (2014) <http://www.healthprofessionals.gov.sg/conten t/hprof/smc/en/leftnav/information_for_register eddoctors.html> [accessed on August 03. 2014].

Stephens, M. B., McKenna, M. \& Carrington, K. (2011) Adult Learning Models for Large-group Continuing Medical Education Activities, Family Medicine, 43,5, pp 334-7.

World Federation for Medical Education (WFME). Continuing Professional Development (CPD) of medical doctors. WFME global standards for quality improvement(2003) www.wfme.org/standards/cpd/.../16continuing-professional-development [accessed on March 05. 2013].

World Health Organization (WHO). Regional guidelines for continuing medical education (CME) / continuing professional development (CPD) activities. (2010) https://www.wbginvestmentclimate.org/toolkits/ health-in-africa-policy-toolkit/upload/WHOCME-Requirements.pdf_ [accessed on May 